\title{
Reconstructing the density and temperature structure of prestellar cores from Herschel data: A case study for B68 and L1689B ${ }^{\star}$
}

\author{
A. Roy ${ }^{1}$, Ph. André ${ }^{1}$, P. Palmeirim ${ }^{1}$, M. Attard ${ }^{1}$, V. Könyves ${ }^{1,2}$, N. Schneider ${ }^{1,3}$, N. Peretto ${ }^{1,4}$, A. Men'shchikov ${ }^{1}$, \\ D. Ward-Thompson ${ }^{5}$, J. Kirk ${ }^{5}$, M. Griffin ${ }^{4}$, K. Marsh ${ }^{4}$, A. Abergel ${ }^{2}$, D. Arzoumanian ${ }^{2}$, M. Benedettini' ${ }^{6}$, T. Hill ${ }^{1,7}$, \\ F. Motte ${ }^{1}$, Q. Nguyen Luong ${ }^{8}$, S. Pezzuto ${ }^{6}$, A. Rivera-Ingraham ${ }^{9,10}$, H. Roussel ${ }^{11}$, K. L. J. Rygl ${ }^{6}$, L. Spinoglio ${ }^{6}$, \\ D. Stamatellos ${ }^{5}$, and G. White ${ }^{12,13}$
}

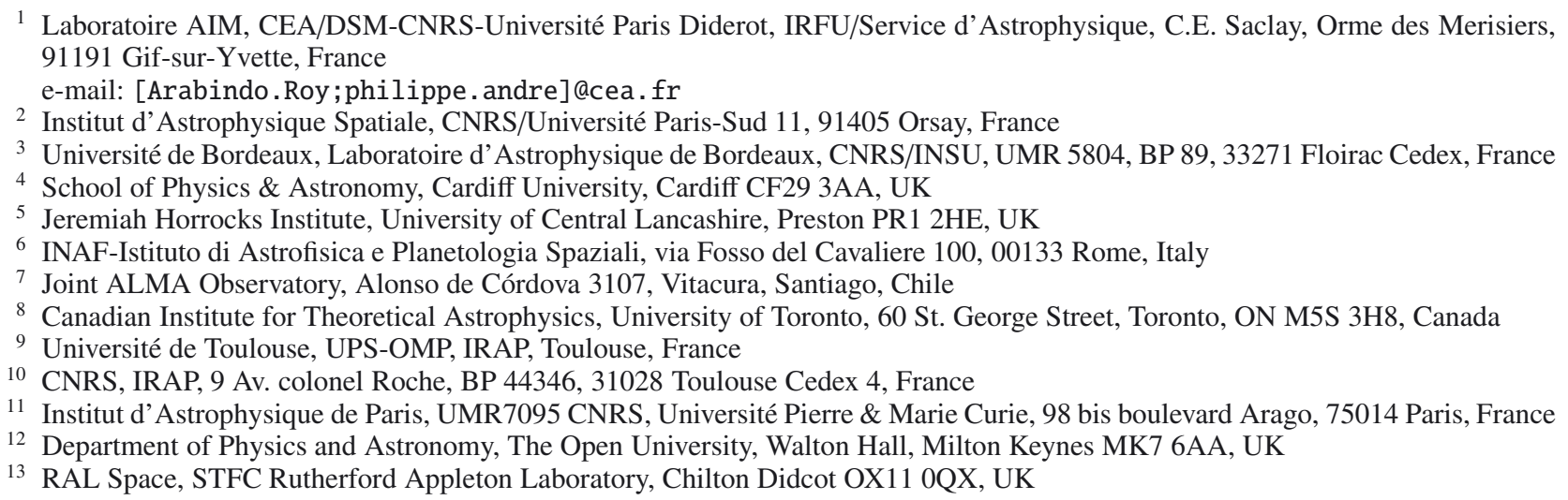

Received 9 July 2013 / Accepted 8 November 2013

\begin{abstract}
Utilizing multiwavelength dust emission maps acquired with Herschel, we reconstruct local volume density and dust temperature profiles for the prestellar cores B68 and L1689B using an inverse-Abel transform-based technique. We present intrinsic radial dust temperature profiles of starless cores directly from dust continuum emission maps disentangling the effect of temperature variations along the line of sight, which were previously limited to the radiative transfer calculations. The reconstructed dust temperature profiles show a significant drop in the core center, a flat inner part, and a rising outward trend until the background cloud temperature is reached. The central beam-averaged dust temperatures obtained for B68 and L1689B are $9.3 \pm 0.5 \mathrm{~K}$ and $9.8 \pm 0.5 \mathrm{~K}$, respectively, which are lower than the temperatures of $11.3 \mathrm{~K}$ and $11.6 \mathrm{~K}$ obtained from direct SED fitting. The best mass estimates derived by integrating the volume density profiles of B68 and L1689B are $1.6 M_{\odot}$ and $11 M_{\odot}$, respectively. Comparing our results for B68 with the near-infrared extinction studies, we find that the dust opacity law adopted by the HGBS project, $\kappa_{\lambda}=0.1 \times\left(\frac{\lambda}{300 \mu \mathrm{m}}\right)^{-2} \mathrm{~cm}^{2} \mathrm{~g}^{-1}$ agrees to within $50 \%$ with the dust extinction constraints.
\end{abstract}

Key words. stars: formation - submillimeter: general - ISM: individual objects: B68 - ISM: individual objects: L1689B dust, extinction

\section{Introduction}

Recent submillimeter observations with the Herschel Space Observatory (Pilbratt et al. 2010) and particularly the results obtained as part of the Herschel Gould Belt Survey (HGBS; André et al. 2010) have significantly improved our global understanding of the early stages of low-mass star formation. It is now becoming clear that the formation of prestellar cores is intimately related to the ubiquitous filamentary structure present in the cold interstellar medium (ISM; Arzoumanian et al. 2011). On one hand one of the main objectives of the HGBS is to measure the prestellar core mass function (CMF) in nearby cloud complexes and to clarify the relationship between the CMF and the stellar

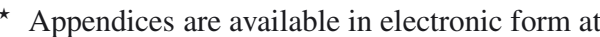
http://www . aanda.org
}

initial mass function (IMF) on the other hand we also look at the link with the structure of the ISM (cf. Könyves et al. 2010; André et al. 2010, for preliminary results).

An accurate determination of the prestellar $\mathrm{CMF}$ requires reliable estimates of core masses. In the context of the HGBS project, core masses are derived from dust continuum emission maps obtained with Herschel between $160 \mu \mathrm{m}$ and $500 \mu \mathrm{m}$. Dust emission is almost always optically thin at these wavelengths and can thus act as a surrogate tracer of the total (gas + dust) mass along the line of sight (LOS). This requires an assumption about the dust opacity in the submillimeter regime and reliable estimates of the dust temperature, $T_{\mathrm{d}}$. Herschel multiwavelength data can be used to estimate $\left\langle T_{\mathrm{d}}\right\rangle_{\mathrm{LOS}}$ through single-temperature greybody fits to the observed spectral energy distributions (SEDs; cf. Könyves et al. 2010). A complication, 
however, is that such fits only provide the average temperature along the LOS and do not account for temperature gradients within the target sources. This is potentially a very significant problem when studying self-gravitating starless (or protostellar) cores with stratified density structures heated by an external or internal radiation field. The central temperatures of cold, starless cores and sometimes their mass-averaged temperatures can be overestimated due to the relatively strong emission from the screen of warmer dust in the outer layers of the cores which bias core mass estimates to lower values (cf. Malinen et al. 2011). Likewise, temperature variations along the LOS may hamper the derivation of reliable density profiles for prestellar cores (e.g., Kirk et al. 2005; Ysard et al. 2012) using submillimeter emission maps (e.g., Kirk et al. 2005; Ysard et al. 2012). Radiative transfer calculations (e.g., Evans et al. 2001; Stamatellos et al. 2007) have been performed to predict the dust temperature profiles of starless cores whose outer surfaces are exposed to heating by the local interstellar radiation field (ISRF). These calculations generally find a significant drop in $T_{\mathrm{d}}$ at the center of starless cores, but the actual magnitude of this temperature drop remained poorly constrained observationally before the advent of Herschel (see Ward-Thompson et al. 2002 for early results with ISO, however).

Here, we use Herschel observations to quantify the dust temperature gradient within two well-studied starless cores, B68 and L1689B. We introduce a simple yet powerful inversion technique based on the Abel integral transform to simultaneously reconstruct the 3D density and dust temperature profiles of dense cores by using the inputs of Herschel maps in four bands between $160 \mu \mathrm{m}$ and $500 \mu \mathrm{m}$ (also see Marsh et al. 2014 for an independent approach of solving similar problem). The basic goal of the present paper is to demonstrate the performance of the Abel inversion technique. We apply our algorithm to B68 and L1689B and compare the results with previous infrared extinction/absorption studies, which allow us to validate the dust opacity assumption made in earlier HGBS papers. We also test our technique on synthetic core models with known density and temperature profiles. While the method assumes spherically symmetric cores, we show that it yields satisfactory results for prolate or oblate ellipsoidal cores with realistic aspect ratios that are $\lesssim 2$.

\section{Herschel observations of B68 and L1689B}

As part of the HGBS key project, two fields in the Pipe and Ophiuchus molecular cloud complexes containing B68 and L1689B with areas of $\sim 1.5 \times 1.5$ and $\sim 3.0 \times 3.5$ were observed for an integration time of 2.6 and $10.2 \mathrm{~h}$, respectively. These target fields were mapped ${ }^{1}$ in two orthogonal scan directions at a scanning speed of $60^{\prime \prime} \mathrm{s}^{-1}$ in parallel mode, acquiring data simultaneously in five bands with the SPIRE (Griffin et al. 2010) and PACS (Poglitsch et al. 2010) bolometer cameras. The data were reduced using HIPE version 7.0. The SPIRE data were processed with modified pipeline scripts. Observations during the turnaround of the telescope were included, and a destriper module with a zero-order polynomial baseline was applied. The default "naïve" mapper was used to produce the final map. For the PACS data, we applied the standard HIPE data reduction

\footnotetext{
1 A more detailed description about the observations and data reductions are available on the HGBS archives webpage: http://gouldbelt-herschel.cea.fr/archives. The reduced SPIRE/PACS maps for B68 and L1689B as well as the column density and temperature maps can also be retrieved from the same website.
}

pipeline up to level 1 with improved calibration. Further processing of the data, such as subtraction of (thermal and non-thermal) low-frequency noise and map projection was performed with Scanamorphos v11 (Roussel 2012). Note that the Scanamorphos map-maker avoids any high-pass filtering, which is crucial for preserving extended emission.

\section{Description of the Abel inversion method}

Consider a spherically symmetric core with radial density profile, $\rho(r)$, embedded in a uniform background and isotropic ISRF. Assuming optically thin dust emission, the specific intensity $I_{v}(p)$ of the core when observed at impact parameter, $p$, may be expressed as

$I_{\nu}(p)=2 \int_{p}^{+\infty} \rho(r) B_{v}\left[T_{\mathrm{d}}(r)\right] \kappa_{v} \frac{r \mathrm{~d} r}{\sqrt{r^{2}-p^{2}}}+I_{v, \mathrm{bg}}+I_{v, \mathrm{~N}}$,

where $I_{v, \text { bg }}$ and $I_{v, \mathrm{~N}}$ represent the background emission and instrumental noise, respectively, $B_{v}\left[T_{\mathrm{d}}(r)\right]$ is the Planck function for the dust temperature $T_{\mathrm{d}}(r)$ at radius $r$ from core center, and $\kappa_{v}$ is the frequency-dependent dust opacity ${ }^{2}$, which is assumed here to be uniform throughout the core. Given the symmetry of the problem, we can use the inverse Abel transform (e.g. Bracewell 1986) to obtain the integrand of Eq. (1) at each observed frequency $v$ :

$\rho(r) B_{v}\left[T_{\mathrm{d}}(r)\right] \kappa_{v}=-\frac{1}{\pi} \int_{r}^{+\infty} \frac{\mathrm{d} I_{v}}{\mathrm{~d} p} \frac{\mathrm{d} p}{\sqrt{p^{2}-r^{2}}}$.

From Eq. (2), we see that the physical parameters of interest, $\rho(r)$ and $T_{\mathrm{d}}(r)$, only depend on the first derivatives of the radial intensity profiles. With a pre-defined assumption about the dust opacity law $\kappa_{v}$, one may thus estimate $T_{\mathrm{d}}(r)$ at each radius $r$ by fitting a single-temperature modified blackbody to the SED obtained from evaluating the right-hand side of Eq. (2) at each observed band between $160 \mu \mathrm{m}$ and $500 \mu \mathrm{m}$ (see Sect. 3.1 for further details). The density profile, $\rho(r)$, can be derived simultaneously from the normalization of the fit at each radius.

For simplicity, Eq. (1) above neglects the convolution with the telescope beam. However, simulations confirm that beam smearing has little effect in the case of well-resolved cores (see Appendix B). In the case of starless cores, such as B68 and L1689B, with a flat inner density profile inside a radius $R_{\text {flat }}$, or angular radius $\theta_{\text {flat }}$, we find that the beam effect can be parameterized by the ratio $\theta_{\text {flat }} / H P B W$ (where $\mathrm{HPBW}$ is the halfpower beam width), and that the reconstructed column density profile agrees with the intrinsic profile within $20 \%$ (in the absence of noise) for $\theta_{\text {flat }} / H P B W \gtrsim 1$ as is the case for B68 and L1689B. More generally, simulations indicate that the reconstructed temperature and column density profiles essentially coincide with the corresponding intrinsic profiles convolved to the effective beam resolution (see Appendix B) in the absence of noise. Importantly, our Abel inversion technique does not depend on the subtraction of a flat background level, since the derivative of a constant background ${ }^{3}$ does not contribute to the integral of Eq. (2). The reconstruction is, however, quite sensitive to noise fluctuations in the outer parts of the core.

\footnotetext{
2 Note the dust-to-gas fraction of $1 \%$ is implicitly included in our definition of the dust opacity (see also Sect. 3.1) so that $\rho(r)$ represents the radial gas density of the object.

3 If the background is not flat, the input data can be pre-processed by subtracting e.g., a linear plane.
} 


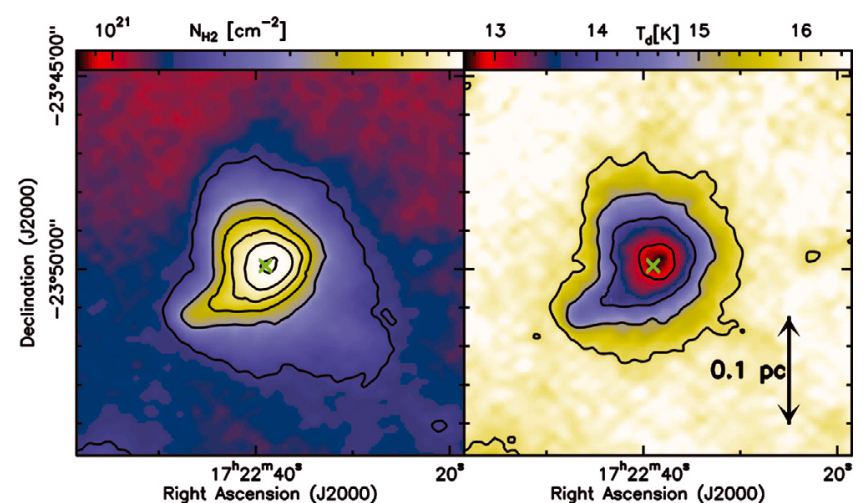

Fig. 1. Column density map (left) and LOS dust temperature map (right) of B68, which is derived by fitting modified blackbody SEDs to the Herschel data between $160 \mu \mathrm{m}$ and $500 \mu \mathrm{m}$ on a pixel-bypixel basis (see Sect. 3.1 for details). The column density contours go from $1.6 \times 10^{21}$ to $9.6 \times 10^{22}$ in steps of $1.6 \times 10^{21}$ in units of $\mathrm{H}_{2}$ molecules per $\mathrm{cm}^{2}$ (left), and the temperature contours are 16, 15, 14 , and $13 \mathrm{~K}$ (right). The cross symbol shows the center of the core obtained by fitting a 2D-Gaussian to the column density map.

Table 1. Planck offsets in $\mathrm{MJy} \mathrm{sr}^{-1}$.

\begin{tabular}{lcccc}
\hline \hline Target & $160 \mu \mathrm{m}$ & $250 \mu \mathrm{m}$ & $350 \mu \mathrm{m}$ & $500 \mu \mathrm{m}$ \\
\hline B68 & 91.2 & 77.0 & 41.6 & 16.6 \\
L1689B & 89.3 & 136.3 & 64.4 & 26.6 \\
\hline
\end{tabular}

\subsection{SED fitting and profile reconstruction}

Column density and corresponding LOS-averaged dust temperature maps are shown in Figs. 1 and 3 for B68 and L1689B, respectively. They were obtained by fitting modified blackbody functions to the Herschel SEDs longward of $160 \mu \mathrm{m}$ on a pixelby-pixel basis, as seen in earlier HGBS papers (Könyves et al. 2010; see also Hill et al. 2011 for HOBYS). While fitting the SEDs, we weighted each data point by the corresponding calibration errors at SPIRE $(\sim 10 \%)$ and PACS $(\sim 15 \%)$ wavelengths. Appropriate zero-level offsets were added to each image (see Table 1), which were obtained by correlating the Herschel data with the Planck and IRAS data of the same fields (see Bernard et al. 2010). The same dust opacity law as in earlier HGBS (see also Motte et al. 2010 for the HOBYS key program) papers is adopted in the present paper (similar to Hildebrand 1983): $\kappa_{\lambda}=0.1 \times\left(\frac{\lambda}{300 \mu \mathrm{m}}\right)^{-\beta} \mathrm{cm}^{2}$ per $\mathrm{g}$ (of gas + dust) with a dust emissivity index of $\beta=2$. Our dust opacity value at the normalizing wavelength is also close to the Ossenkopf \& Henning (1994) opacity model for dust grains with thin ice mantles. A mean molecular weight $\mu_{\mathrm{H}_{2}}=2.8$ is assumed ${ }^{4}$ to express column density in units of $\mathrm{H}_{2}$ molecules per $\mathrm{cm}^{2}$. Using our adopted dust opacity law, we find that even the central LOSs for B68 and L1689B with $N_{\mathrm{H}_{2}} \leq 5 \times 10^{22} \mathrm{~cm}^{-2}$ has small optical depths $\lesssim 0.08$ at $160 \mu \mathrm{m}$, confirming that the core emission is optically thin longward of $160 \mu \mathrm{m}$.

The same assumptions have been adopted for the modified blackbody fits required at each radius by the Abel inversion technique. A single-temperature description of the SEDs is suitable in this case because we are sampling both local density and

\footnotetext{
4 Note that this differs from the first HGBS papers (e.g. André et al. 2010; Könyves et al. 2010; Arzoumanian et al. 2011), where $\mu=2.33$ was assumed and column density was expressed in units of mean free particles per $\mathrm{cm}^{2}$.
}

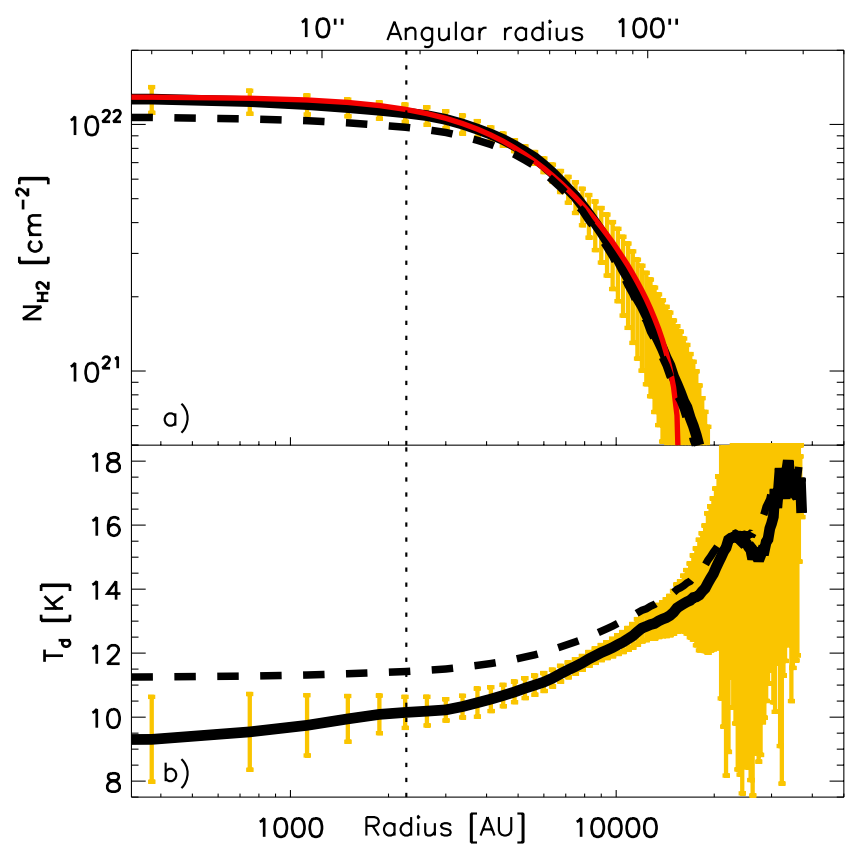

Fig. 2. Column density a) and dust temperature b) profiles of B68 obtained at $500 \mu \mathrm{m}$ resolution by applying the Abel inversion method to the circularly averaged intensity profiles observed with Herschel between $160 \mu \mathrm{m}$ and $500 \mu \mathrm{m}$. a) Comparison between the column density profiles derived from the Abel reconstruction (solid black line) and LOS-averaged SED fitting (thick dashed line). The red curve shows the best-fit Bonnor-Ebert model to the Abel-inverted profile (see Table 2 for parameters). The vertical dotted line represents the half power beam radius of 36.' $3 / 2$ (effective $500 \mu \mathrm{m}$ resolution). b) Comparison of the Abel-inverted (solid line) and LOS-averaged SED (dashed curve) temperature profiles. The error bars represent the standard deviation of $N_{\mathrm{H}_{2}}(r)$ and $T_{\mathrm{d}}(r)$ values obtained from independent profile reconstructions along sixteen angular directions.

dust temperature at a given radius $r$. Prior to the SED fitting, the Herschel data are convolved to a common resolution of either 36 ". 3 (i.e., HPBW resolution of SPIRE at $500 \mu \mathrm{m}$ ) or $24 . ' 9$ (i.e., HPBW resolution of SPIRE at $350 \mu \mathrm{m}$ when the $500 \mu \mathrm{m}$ data are not used). At each wavelength, a circularly averaged intensity profile about the core center is first derived from the Herschel data, and the derivative of this average profile is then numerically evaluated and integrated over the kernel shown in the right-hand side of Eq. (2). The central position of the core is obtained from fitting a 2D-Gaussian model to the column density map (see the cross symbols in Figs. 1 and 3 for B68 and L1689B, respectively). The integration is performed up to an outer radius corresponding to $\sim 2-2.5$ times the FWHM diameter of the core as estimated from the 2D-Gaussian fit to the column density map. In practice, the integral on the right-hand side of Eq. (2) converges rapidly and does not depend much on the precise value of the integration outer radius as long as it encompasses the entire core - see Appendix A and Fig. A.1.

\subsection{Assessment of uncertainties}

The errors bars on the reconstructed profiles at each radius can be estimated from the standard deviations of the density and temperature profiles obtained by repeating the Abel reconstruction along different angular directions around the source (i.e., averaging the data separately over a series of angular sectors instead of circularly averaging the data). In the case of the reconstructed profiles of B68 and L1689B as shown in Figs. 2 and 4, 


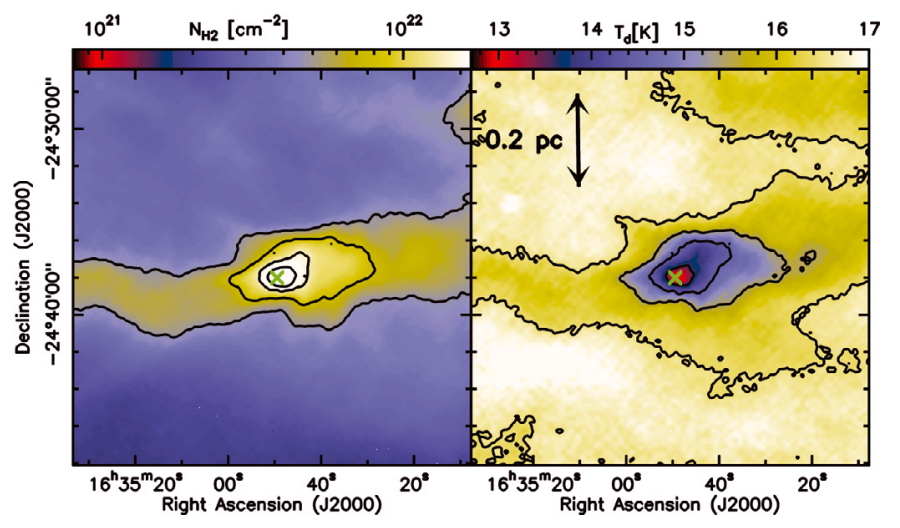

Fig. 3. Same as Fig. 1 but for L1689B. The column density contours are $6 \times 10^{21}, 1 \times 10^{22}, 1.4 \times 10^{22}, 1.8 \times 10^{22}$, and $2.6 \times 10^{22} \mathrm{H}_{2} \mathrm{~cm}^{-2}$ (left), and the temperature contours are 16.5, 15.5, 14.5, and $13.5 \mathrm{~K}$ (right). The column density image shows that L1689B is embedded inside a filamentary structure.

sixteen equally-spaced angular sectors were used to derive the error bars. The errors we report on the best estimates of the (column) density and temperature at core center correspond to the standard error in the mean, $\sigma / \sqrt{n}$, where $n$ is the number of independently measured sectors.

The uncertainty in the normalization of the dust opacity law directly affects the column density estimates but not the temperature estimates, whereas the uncertainty in the emissivity index $\beta$ influences both the dust temperature and the column density estimates in an anti-correlated fashion. When $\beta$ is varied from 2 to 1.5 , for instance, the dust temperature increases by $\sim 10 \%$ and the column density decreases by $\sim 40 \%$. The uncertainty in the central position of the core also introduces errors on the derived parameters. To assess the magnitude of this effect in the case of B68, we varied the central position within a radius of $6^{\prime \prime}$ and found that the resulting central dust temperature and central column density had standard deviations of $\pm 0.3 \mathrm{~K}$ and $\pm 0.04 \times 10^{22} \mathrm{~cm}^{-2}$, respectively. Additionally, departures from spherical symmetry due to asymmetries in the density distribution (cf. L1689B in Fig. 3) or an anisotropic background radiation field (cf. Nielbock et al. 2012) may break the symmetry of the quantity $\rho(r) B_{v}(T(r))$ in Eqs. (1) and (2), introducing additional errors. In principle the latter are included in the error bars estimated from the fluctuations of the radial profiles reconstructed along various angular directions. Moreover, we show in Appendix B that cores with moderate departures from spherical symmetry (such as prolate or oblate ellipsoidal cores with aspect ratios $\$ 2$ ) can be reconstructed with reasonable accuracy.

For both B68 and L1689B, the net measurement errors in the derived central $T_{\mathrm{d}}$ and $N_{\mathrm{H}_{2}}$ values are estimated to be $\pm 0.5 \mathrm{~K}$ and $\pm 0.1 \times 10^{22} \mathrm{~cm}^{-2}$, respectively, excluding the systematic uncertainties associated with our assumptions on the dust opacity and the calibration errors. In Appendix B.1 we show that the calibration errors lead to an additional uncertainty of $\sim 12 \%$ on column density and $\sim 5 \%$ on temperature estimates.

\section{Detailed results for B68 and L1689B}

\subsection{B68 core}

B68 is a well studied isolated bok globule in the Pipe nebula cloud complex (e.g. Alves et al. 2001; Nielbock et al. 2012). We adopt a distance of 125 pc (de Geus et al. 1989) for the present study. Figure 2 shows the reconstructed column density and temperature profiles obtained from our Herschel observations of B68 with the Abel inversion method described as in Sect. 3.

In Fig. 2b, the Abel-inverted radial dust temperature profile (solid curve) is compared with the LOS-averaged SED temperature profile (dashed curve). While both temperature profiles exhibit similar features with a broad minimum around the core center, a positive gradient outside the flat inner plateau of the column density profile, and similar values $\left(T_{\mathrm{d}} \sim 16.5 \pm 2 \mathrm{~K}\right)$ at large radii, the minimum Abel-reconstructed temperature at core center is $\sim 2 \mathrm{~K}$ lower than the minimum SED temperature observed through the central LOS. Accordingly, the central column density derived with the Abel inversion method $\left(1.3 \pm 0.1 \times 10^{22} \mathrm{~cm}^{-2}\right)$ is $30 \%$ higher than the LOS-averaged column density of $\sim 1.0 \times 10^{22} \mathrm{~cm}^{-2}$ that is derived from standard SED fitting for the central LOS. The difference between the Abel-reconstructed and the LOS-averaged column density becomes negligible in the outer parts of the core. This is indicative of stronger temperature variations along the central LOS compared to the outer LOSs.

Recently, Nielbock et al. (2012) constrained the dust temperature and volume density profiles of B68 using an iterative approach based on 3D radiative transfer modeling of multiwavelength dust continuum data. This included Herschel observations that were obtained as a part of the EPoS (Launhardt et al. 2013) key project. They employed a 3-dimensional grid of Plummerlike (Plummer 1911) density profiles and obtained initial guesses of the parameters from LOS-averaged SED fits. Altogether their model was tuned with eight free parameters, yielding a central dust temperature of $T_{\mathrm{d}}=8.2_{-0.7}^{+2.1} \mathrm{~K}$. The primary reason for the dispersion in the central dust temperature in Nielbock et al. (2012) is the uncertainty of a factor of $\sim 2$ on the dust opacity in the infrared regime. Although we obtain a higher central dust temperature, $T_{\mathrm{d}}=9.3 \pm 0.5 \mathrm{~K}$, with our Abel-inversion method, our results agree with the Nielbock et al. analysis within the range of the quoted uncertainties.

Based on extinction measurements (Alves et al. 2001), the column density profile of B68 closely resembles that of a Bonnor-Ebert (BE; e.g., Bonnor 1956) isothermal sphere with a flattened inner region. The overplotted red curve in Fig. 2 shows the best-fit BE model to the reconstructed column density profile. The best-fit BE parameters along with the physical properties that follow directly from the fit such as the radius of the flat inner plateau, $R_{\text {flat }} \equiv 2 \mathrm{c}_{\mathrm{s}} / \sqrt{4 \pi G \rho_{\mathrm{c}}}$, the density contrast, $\rho_{\mathrm{c}} / \rho_{\mathrm{s}}$, and the external pressure, $P_{\text {ext }}$, are summarized in Table 2. They are consistent with the BE parameters found by Alves et al. (2001).

The $N_{\mathrm{H}_{2}}$ column density profile obtained by integrating the Abel-inverted volume density profile is shown in Fig. 2. This can be directly compared with the near-infrared extinction results Alves et al. (2001) because extinction traces material independently of temperature. For this comparison, we adopted a standard conversion factor, $N_{\mathrm{H}_{2}} / A_{\mathrm{V}}$ of $9.4 \times 10^{20} \mathrm{~cm}^{-2} \mathrm{mag}^{-1}$ (Bohlin et al. 1978), to translate column density to equivalent visual extinction $A_{\mathrm{V}}$ at low column densities (i.e., $N_{\mathrm{H}_{2}} \lesssim$ $6 \times 10^{21} \mathrm{~cm}^{-2}$ ). For higher column densities, we used the conversion $N_{\mathrm{H}_{2}} / A_{\mathrm{V}}=6.9 \times 10^{20} \mathrm{~cm}^{-2} \mathrm{mag}^{-1}$ given by Draine (2003) and later adopted by Evans et al. (2009), which is consistent with an extinction curve with a total-to-selective extinction, $R_{\mathrm{V}} \equiv A_{\mathrm{V}} / E(B-V)=5.5$ that is appropriate in higher density regions.

Using the Bohlin conversion factor, we obtain an $A_{\mathrm{V}}$ of $3.2 \mathrm{mag}$ at a radius of $10^{4} \mathrm{AU}$, which agrees within $\sim 60 \%$ with the value of 5 mag reported by Alves et al. (2001) at the 
A. Roy et al.: Reconstructing density and temperature profiles

Table 2. Best-fit parameters of Bonnor-Ebert core models for B68 and L1689B.

\begin{tabular}{lrrccccc}
\hline \hline $\begin{array}{l}\text { Source } \\
\text { profile }\end{array}$ & $\xi_{\max }$ & $\rho_{\mathrm{c}} / \rho_{\mathrm{s}}{ }^{a}$ & $\begin{array}{c}\mathrm{c}_{s}{ }^{b} \\
\left(\mathrm{~km} \mathrm{~s}^{-1}\right)\end{array}$ & $\begin{array}{c}P_{\text {ext }} \\
\left(\mathrm{K} \mathrm{cm}^{-3}\right) \\
\times 10^{4}\end{array}$ & $\begin{array}{c}R_{\text {flat }}{ }^{c} \\
(\mathrm{AU}) \\
\times 10^{3}\end{array}$ & $\begin{array}{c}R_{\text {out }} \\
(\mathrm{AU})\end{array}$ & $\begin{array}{c}n_{\mathrm{c}} \\
\left(\mathrm{cm}^{-3}\right) \\
\times 10^{4}\end{array}$ \\
\hline B68 & $7.0 \pm 0.1$ & 17 & 0.17 & 3.9 & 4.4 & 1.5 & 8.3 \\
L1689B-C & $16.6 \pm 0.7$ & 145 & 0.27 & 2.9 & 4.5 & 3.7 & 20 \\
L1689B-NS & $13.5 \pm 0.3$ & 88 & 0.24 & 3.2 & 4.4 & 2.9 & 17 \\
L1689B-EW & $16.2 \pm 1.7$ & 137 & 0.26 & 2.2 & 4.7 & 3.8 & 16.5 \\
\hline
\end{tabular}

Notes. ${ }^{(a)} \rho_{\mathrm{c}}$ and $\rho_{\mathrm{S}}$ denote central density and density at the outer surface of the BE sphere, respectively. ${ }^{(b)}$ Isothermal sound speed. ${ }^{(c)}$ Flat inner radius, defined as $R_{\text {flat }} \equiv 2 c_{\mathrm{s}} / \sqrt{4 \pi G \rho_{\mathrm{c}}}$.

same radius. Furthermore, if we correct our estimate for the weak empirical trend between submillimeter dust opacity and column density, $\kappa_{\lambda} \propto N_{\mathrm{H}_{2}}{ }^{0.28}$, as inferred by Roy et al. (2013) in the regime of $1 \lesssim A_{V} \lesssim 10$ and interpreted as evidence of dust grain evolution, then we find a corrected column density $N_{\mathrm{H}_{2}}^{\text {corr }} \approx 5 \times 10^{21} \mathrm{~cm}^{-2}$, which is equivalent to a corrected $A_{V}^{\text {corr }}=5.3 \mathrm{mag}$. This is in excellent (10\%) agreement with Alves et al. (2001) at $10^{4} \mathrm{AU}$.

Using the Draine conversion factor, we derive an $A_{\mathrm{V}}$ of $19 \pm$ 2 mag through the center of the B68 core as compared to the $A_{\mathrm{V}}$ of 30 mag obtained from extinction by Alves et al. (2001), corresponding to a $60 \%$ agreement. However, the effective angular resolution of the Alves et al. extinction map was about $\sim 10^{\prime \prime}$, approximately four times higher than the beam resolution (36.' 3 ) of SPIRE at $500 \mu \mathrm{m}$. For better comparison, we performed a similar Abel reconstruction analysis at the beam resolution (24.'9) of the SPIRE $350 \mu \mathrm{m}$ observations, ignoring the $500 \mu \mathrm{m}$ data. In this case, the central Abel-reconstructed column density corresponds to $A_{V}=20 \pm 2 \mathrm{mag}$, which agrees within $50 \%$ with the results of Alves et al. (2001). (The central dust temperature derived at $350 \mu \mathrm{m}$ resolution coincides within the uncertainties with the $500 \mu \mathrm{m}$ resolution estimate.)

Likewise, the total mass of $1.6 \pm 0.07 M_{\odot}$, which is derived by integrating the Abel-reconstructed density profile of $\mathrm{B} 68$ within the outer radius of $1.4 \times 10^{4} \mathrm{AU}$, agrees within $30 \%$ with the mass of $2.1 M_{\odot}$ as obtained by Alves et al. (2001) from the extinction data. For comparison, the mass derived from fitting a modified blackbody to the integrated flux densities is $1.4 \pm 0.05 M_{\odot}$. The latter does not account for temperature variations along the LOS and thus slightly underestimates the intrinsic total mass of the core. For a moderate density core such as B68, however, we stress that the global SED temperature $(12.5 \pm 0.1 \mathrm{~K}$ here) is close to the mass-averaged dust temperature and the SED mass agrees within $\sim 15 \%$ of the Abel-reconstructed mass.

Using the above results on the density and temperature structure, we can check the energy balance of the B68 core. Assuming optically thin submillimeter emission, a total output luminosity of $0.35 \pm 0.04 L_{\odot}$ is obtained by integrating the quantity $4 \pi \int \rho(r) B_{v}\left[T_{\mathrm{d}}(r)\right] \kappa_{v} \mathrm{~d} v$ over the volume of the core. A very similar output luminosity $\left(0.41 \pm 0.05 L_{\odot}\right)$ is estimated by integrating the observed SED over wavelengths. These output estimates should be compared to the input luminosity of $\sim 0.40 \mathrm{~L}_{\odot}$ provided to the core by the local ISRF (Mathis et al. 1983; with $G_{0} \sim 1$ ), which are calculated from the total ISRF flux density that is absorbed ${ }^{5}$ by a spherical object with the same density

\footnotetext{
5 The ISRF energy is mostly absorbed at short wavelengths $(0.095 \mu \mathrm{m}$ to $100 \mu \mathrm{m})$. In calculating the absorbed energy we adopted the dust absorption model of Draine (2003) with $R_{\mathrm{V}}=3.1$.
}

profile and outer radius as B68 using Eq. (4) of Lehtinen et al. (1998). Note that the three luminosity values above agree with one another.

\section{2. $L 1689 B$}

Compared to B68, L1689B is a slightly denser and more centrally condensed core (e.g. André et al. 1996; Bacmann et al. 2000) located in the Ophiuchus complex at a distance of $\sim 140 \mathrm{pc}$. The Herschel images reveal that it is embedded within a larger-scale filamentary structure (see Fig. 3). Given the elongated morphology that is observed in the plane of the sky and the mean apparent aspect ratio $\sim 1.3$ of the core, the hypothesis of spherical symmetry underlying Eq. (1) is not strictly verified.

To assess the validity of our Abel-inversion scheme in this case, we therefore performed test reconstructions for prolate and oblate ellipsoidal model cores with similar aspect ratios. These tests suggest that small departures from spherical symmetry have little impact on the reconstruction results (see Appendix B).

The upper panel of Fig. 4 shows the results of three distinct reconstructions of the column density profile of L1689B, as obtained by applying the Abel inversion method to a) the circularly averaged intensity profiles (thick solid curve); b) the intensity profiles observed in sectors oriented east-west (EW), i.e., approximately along the apparent major axis of the core (blue dash-dotted curve); and c) the intensity profiles observed in sectors oriented north-south (NS), i.e., approximately along the apparent minor axis of the core (green dash-dotted curve). While the three column density profiles agree with each other within the central plateau region, the EW profile lies $30 \%$ above the NS profile at large radii $\gtrsim 10^{4} \mathrm{AU}$, and the circularly averaged profile is intermediate between the other two. For reference and comparison with B68, a BE model was fitted to each of the three column density profiles and the results of these fits are given in Table 2. (For the sake of clarity, only the model fit to the circularly averaged intensity profiles is shown in Fig. 4, as a red solid curve.) All three fits yield a consistent value for the radius of the flat inner plateau, $R_{\text {flat }} \sim 4500 \pm 100$ AU (corresponding to $\left.\sim 32^{\prime \prime}\right)$. The average reconstructed column density within $R_{\text {flat }}$ is $3.5 \pm 0.1 \times 10^{22} \mathrm{~cm}^{-2}$, which agrees well $(\sim 30 \%)$ with the $\mathrm{H}_{2}$ column density that is averaged over the flat inner part of the core of $4.5-4.7 \times 10^{22} \mathrm{~cm}^{-2}$. This value was found by Bacmann et al. (2000) based on their ISOCAM mid-infrared absorption study (see their Table 2).

Our best estimate of the total core mass obtained from Abel reconstructing is $11 \pm 2 M_{\odot}$. The tests we performed for ellipsoidal synthetic cores (cf. Appendix B) indicate that the relative error in this mass introduced by the departure from spherical 


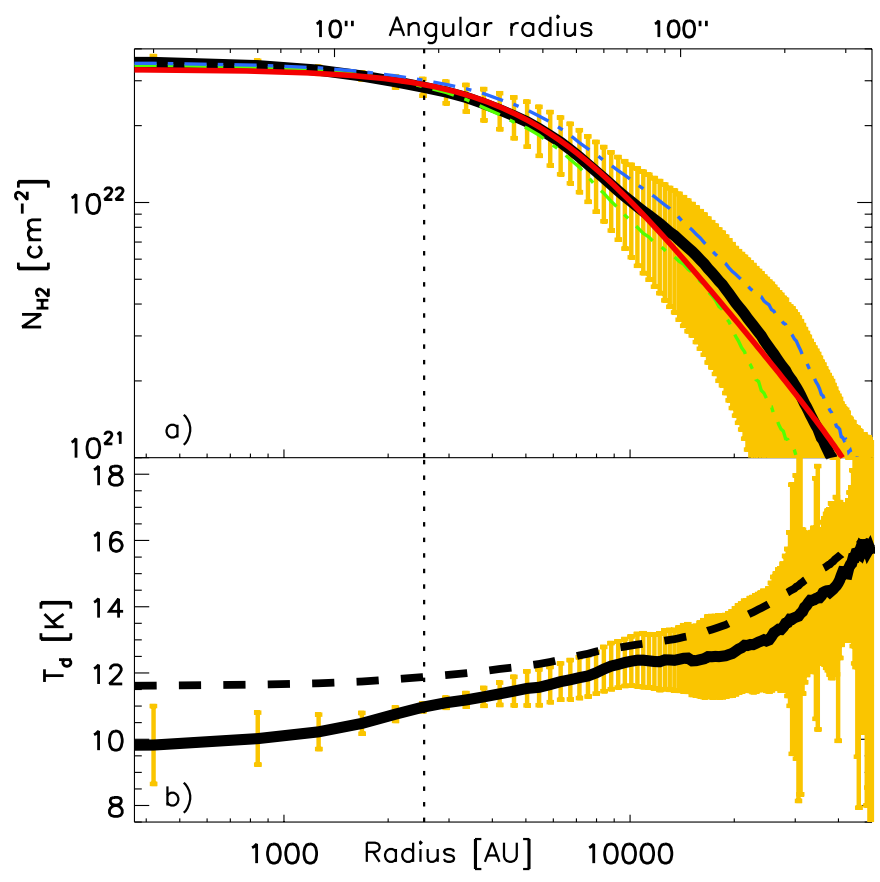

Fig. 4. a) Column density profile of L1689B obtained at $500 \mu \mathrm{m}$ resolution by applying the Abel reconstruction method to the circularly averaged intensity profiles between $160 \mu \mathrm{m}$ and $500 \mu \mathrm{m}$ (solid black curve). The dash-dotted blue and green profiles show the column density profiles obtained from reconstructing the intensity profiles observed along EW and NS sectors, respectively. The overplotted solid red line shows the best-fit Bonnor-Ebert model (see Table 2 for parameters). The vertical dotted line is same as in Fig. 2. b) Reconstructed dust temperature profile of L1689B (solid curve) compared with the LOS-averaged temperature profile derived from simple SED fitting (thick dashed curve).

symmetry is less than $4 \%$ for an intrinsic aspect ratio of $\sim 1.3$. Our mass estimate is in excellent agreement with the total mass reported by Bacmann et al. (2000). The SED mass derived from fitting a modified blackbody to the integrated flux densities is $7.8 \pm 0.2 M_{\odot}$, which is about $30 \%$ lower than our best mass estimate. The corresponding global SED temperature of the core is $12.5 \pm 0.2 \mathrm{~K}$.

The central dust temperature obtained for L1689B using the Abel transform technique is $9.8 \pm 0.5 \mathrm{~K}$ (see lower panel of Fig. 4). This value is higher than the very low central dust temperature of $7.5 \mathrm{~K}$ advocated by Evans et al. (2001) which is based on radiative transfer calculations if a standard ISRF $\left(G_{0}=1\right)$ is assumed. At least part of this discrepancy can be explained by the fact that the effective external radiation field for L1689B is about one order of magnitude stronger than the standard ISRF, due to the presence of early-type stars in the immediate vicinity of the Ophiuchus cloud (cf. Liseau et al. 1999).

\section{Conclusions}

The results of B68 and L1689B discussed in Sect. 4, along with the tests on model cores presented in Appendix B, demonstrate that the Abel transform technique can successfully produce meaningful radial density and dust temperature profiles for spatially resolved prestellar cores. The most rewarding result is the reconstruction of a radial dust temperature profile that is directly from Herschel dust continuum imaging data and independent of any radiative transfer model. The Abel inversion technique (Sect. 3) is very general: it is insensitive to background subtraction to first order and can perform equally well for externally-heated (isotropically) starless cores or internallyheated protostellar cores. While the technique assumes spherically symmetric cores in principle, tests performed on ellipsoidal cores suggest that satisfactory results are obtained even when the hypothesis of spherical symmetry is not strictly valid (such as in the L1689B case - cf. Fig. 3). A similar Abel-transform scheme may also be employed to reconstruct the intrinsic beam-averaged density and temperature profiles of (approximately) cylindrically symmetric filaments from the observed radial intensity profiles averaged along the filament main axes.

For both B68 and L1689B, we find a characteristic dip in the dust temperature profile with minimum beam-averaged values of $9.3 \pm 0.5 \mathrm{~K}$ and $9.8 \pm 0.5 \mathrm{~K}$ at core center, respectively. The temperature profile smoothly merges with the background cloud temperature at the outer core radii.

The Abel transform technique yields central beam-averaged $\mathrm{H}_{2}$ densities of $7.5 \pm 0.5 \times 10^{4} \mathrm{~cm}^{-3}$ and $2.0 \pm 0.1 \times 10^{5} \mathrm{~cm}^{-3}$ for B68 and L1689B, respectively, which correspond to central column densities of $1.3 \pm 0.1 \times 10^{22} \mathrm{~cm}^{-2}$ and $3.6 \pm 0.1 \times 10^{22} \mathrm{~cm}^{-2}$ after integration of the reconstructed volume density profiles along the LOS. These central column density estimates are approximately $15 \%$ larger than the values obtained from direct SED fitting. Comparison of our results with the independent near-IR extinction measurement of the B68 column density profile by Alves et al. (2001) suggests that the dust opacity law adopted by the HGBS consortium, with $\kappa_{300 \mu \mathrm{m}}=0.1 \mathrm{~cm}^{2}$ per $\mathrm{g}$ (of gas + dust) at $\lambda=300 \mu \mathrm{m}$ and $\beta=2$, is more accurate than (and possibly overestimated by) $50 \%$ in the $160-500 \mu \mathrm{m}$ range. This value is for sources of (column) densities that are comparable to B68 and L1689B. Our adopted opacity value is within $\sim 20 \%$ of the value ${ }^{6}$ obtained by Suutarinen et al. (2013) for the dust inside a core of similar column density to the ones considered here. Assuming that the weak trend between submillimeter dust opacity and column density $\left(\kappa_{\lambda} \propto N_{\mathrm{H}_{2}}{ }^{0.28}\right)$ found by Roy et al. (2013) at $A_{V} \lesssim 10$ also holds at higher $A_{V}$, we argue that the HGBS dust opacity law may remain valid to within $50 \%$ accuracy in the whole range of $\mathrm{H}_{2}$ column densities between $\sim 3 \times 10^{21} \mathrm{~cm}^{-2}$ and $\sim 10^{23} \mathrm{~cm}^{-2}$.

Since the Abel transformation technique can help us correct the effect of LOS temperature variations on the derivation of total masses, we conclude that the Herschel data of the HGBS project holds the promise of delivering core masses to accuracy more than factor of 1.5 to 2 , at least for spatially-resolved cores.

Acknowledgements. This work has benefited from the support of the CNES and the European Research Council under the European Union's Seventh Framework Programme (FP7/2007-2013 - ERC Grant Agreement no. 291294). P.P. acknowledges funding by the Fundação para a Ciência e a Tecnologia (Portugal). SPIRE has been developed by a consortium of institutes led by Cardiff Univ. (UK) and including Univ. Lethbridge (Canada); NAOC (China); CEA, LAM (France); IFSI, Univ. Padua (Italy); IAC (Spain); Stockholm Observatory (Sweden); Imperial College London, RAL, UCL-MSSL, UKATC, Univ. Sussex (UK); Caltech, JPL, NHSC, Univ. Colorado (USA). This development has been supported by national funding agencies: CSA (Canada); NAOC (China); CEA, CNES, CNRS (France); ASI (Italy); MCINN (Spain); SNSB (Sweden); STFC (UK); and NASA (USA). PACS has been developed by a consortium of institutes led by MPE (Germany) and including UVIE (Austria); KUL, CSL, IMEC (Belgium); CEA, OAMP (France); MPIA (Germany); IFSI, OAP/AOT, OAA/CAISMI, LENS, SISSA (Italy); IAC (Spain). This development has been supported by the funding agencies BMVIT (Austria), ESA-PRODEX (Belgium), CEA/CNES (France), DLR (Germany), ASI (Italy), and CICT/MCT (Spain).

6 Suutarinen et al. 2013 quote $\kappa_{250 \mu \mathrm{m}}=0.08 \mathrm{~cm}^{2} \mathrm{~g}^{-1}$ with $\beta=2$ and argue that this value is underestimated by $40 \%$ inside the core due to temperature variations along the LOS. 
A. Roy et al.: Reconstructing density and temperature profiles

\section{References}

Alves, J. F., Lada, C. J., \& Lada, E. A. 2001, Nature, 409, 159

André, P., Ward-Thompson, D., \& Motte, F. 1996, A\&A, 314, 625

André, P., Bouwman, J., Belloche, A., \& Hennebelle, P. 2003, in Proc. SFChem

2002: Chemistry as a Diagnostic of Star Formation, eds. C. L. Curry, \&

M. Fich (Ottawa: NRC Press), 127

André, P., Men'shchikov, A., Bontemps, S., et al. 2010, A\&A, 518, L102

Arzoumanian, D., André, P., Didelon, P., et al. 2011, A\&A, 529, L6

Bacmann, A., André, P., Puget, J.-L., et al. 2000, A\&A, 361, 555

Bernard, J.-P., Paradis, D., Marshall, D. J., et al. 2010, A\&A, 518, L88

Bohlin, R. C., Savage, B. D., \& Drake, J. F. 1978, ApJ, 224, 132

Bonnor, W. B. 1956, MNRAS, 116, 351

Bouwman, J., Meeus, G., de Koter, A., et al. 2001, A\&A, 375, 950

Bracewell, R. N. 1986, The Fourier Transform and its applications, 2nd edn. (New York: McGraw-Hill)

de Geus, E. J., de Zeeuw, P. T., \& Lub, J. 1989, A\&A, 216, 44

Draine, B. T. 2003, ARA\&A, 41, 241

Evans, II, N. J., Rawlings, J. M. C., Shirley, Y. L., \& Mundy, L. G. 2001, ApJ, 557,193

Evans, II, N. J., Dunham, M. M., Jørgensen, J. K., et al. 2009, ApJS, 181, 321

Griffin, M. J., Abergel, A., Abreu, A., et al. 2010, A\&A, 518, L3

Hildebrand, R. H. 1983, QJRAS, 24, 267

Hill, T., Motte, F., Didelon, P., et al. 2011, A\&A, 533, A94
Kirk, J. M., Ward-Thompson, D., \& André, P. 2005, MNRAS, 360, 1506 Könyves, V., André, P., Men'shchikov, A., et al. 2010, A\&A, 518, L106 Launhardt, R., Stutz, A. M., Schmiedeke, A., et al. 2013, A\&A, 551, A98 Lehtinen, K., Lemke, D., Mattila, K., \& Haikala, L. K. 1998, A\&A, 333, 702 Liseau, R., White, G. J., Larsson, B., et al. 1999, A\&A, 344, 342

Malinen, J., Juvela, M., Collins, D. C., Lunttila, T., \& Padoan, P. 2011, A\&A, 530, A101

Marsh, K. A., Griffin, M. J., André, P., et al. 2014, MNRAS, submitted [arXiv: 1401.7871]

Mathis, J. S., Mezger, P. G., \& Panagia, N. 1983, A\&A, 128, 212

Men'shchikov, A., André, P., Didelon, P., et al. 2012, A\&A, 542, A81

Motte, F., Zavagno, A., Bontemps, S., et al. 2010, A\&A, 518, L77

Nielbock, M., Launhardt, R., Steinacker, J., et al. 2012, A\&A, 547, A11

Ossenkopf, V., \& Henning, T. 1994, A\&A, 291, 943

Pilbratt, G. L., Riedinger, J. R., Passvogel, T., et al. 2010, A\&A, 518, L1

Plummer, H. C. 1911, MNRAS, 71, 460

Poglitsch, A., Waelkens, C., Geis, N., et al. 2010, A\&A, 518, L2

Roussel, H. 2012, PASP, 125, 1126

Roy, A., Martin, P. G., Polychroni, D., et al. 2013, ApJ, 763, 55

Stamatellos, D., Whitworth, A. P., \& Ward-Thompson, D. 2007, MNRAS, 379 , 1390

Suutarinen, A., Haikala, L. K., Harju, J., et al. 2013, A\&A, 555, A140

Ward-Thompson, D., André, P., \& Kirk, J. M. 2002, MNRAS, 329, 257

Ysard, N., Juvela, M., Demyk, K., et al. 2012, A\&A, 542, A21

Pages 8 to 10 are available in the electronic edition of the journal at http://www . aanda. org 


\section{Appendix A: Surface brightness profiles and the outer radius of $\mathrm{B} 68$}

The top panel of Fig. A.1 shows the circularly averaged intensity profiles of the B68 core at SPIRE and PACS wavelengths, which were used to construct the column density and temperature profiles shown in Fig. 2. The bottom panel of Fig. A.1 shows the logarithmic slope of the column density profile (black line), which is defined as $s \equiv \mathrm{d} \ln N_{\mathrm{H}_{2}} / \mathrm{d} \ln r$ (dimensionless), as a function of radius. The measured logarithmic slope is $s=0$ near the core center due to flat inner density profile and the finite resolution of the observations. The logarithmic slope profile reaches a minimum value $s_{\min } \sim-1.5$ at $r \sim 10^{4} \mathrm{AU}$ and goes back to $s \sim 0$ at the outer boundary, where the core merges with a slowly-varying background. For comparison, a spherical core with outer density profile $\rho \propto r^{-2}$ would have $s=-1$ at large radii. In the same plot, we show the logarithmic slope of the $500 \mu \mathrm{m}$ surface brightness profile (blue line), which has a shallower slope due to the additional effect of the positive outward temperature gradient. Inspection of the intensity and slope profiles shown in Fig. A.1 allows us to select an appropriate upper integration radius in the right-hand side of Eq. (2) when the density and temperature structure of B68 (see Sect. 3) are reconstructed. Since $\frac{\mathrm{d} I_{v}}{\mathrm{~d} p}=s \times \frac{I_{v}}{p} \approx 0$ beyond a radius $\sim 25000 \mathrm{AU}$, larger radii do not contribute to the integral of Eq. (2). In practice, we adopt an upper integration radius $R_{\text {up }}=37500 \mathrm{AU}$ for B68, as shown by the dashed vertical line in Fig. A.1. The reconstruction results, however, are insensitive to the precise choice of $R_{\text {up }}$ as long as $R_{\text {up }} \gtrsim 25000 \mathrm{AU}$. The adjacent dot-dashed vertical line shows the effective radius of $\sim 27000 \mathrm{AU}$ ( or $\sim 200^{\prime \prime}$ ), as derived by the getsources sourcefinding algorithm for the "footprint" of B68. The getsources algorithm (Men'shchikov et al. 2012) is the source extraction method used by the HGBS consortium to produce the firstgeneration catalogs of dense cores found by Herschel in the regions covered by the HGBS survey. The footprint of a core corresponds to the area just outside, of which getsources estimates the local background emission and over which it integrates the background-subtracted emission to derive the total flux densities of the core. In the case of B68, the results automatically derived by getsources are in excellent agreement with those obtained through a detailed radial profile analysis (cf. Fig. A.1).

\section{Appendix B: Tests of the Abel inversion method using simple models}

\section{B.1. Spherically symmetric core model}

To test the performance level of our Abel inversion scheme and quantify the robustness of the reconstruction, we applied the algorithm as described in Sect. 3 to synthetic images corresponding to model starless cores of known density and temperature distributions. First, we considered a spherically-symmetric core model with a Plummer-type density distribution for $r \leq R_{\text {out }}$,

$\rho(r)=\frac{\rho_{\mathrm{c}}}{1+\left(r / R_{\mathrm{flat}}\right)^{2}}$,

which is parameterized by physical parameters that are approximately similar to the derived properties of B68 (see Sect. 4.1): central $\mathrm{H}_{2}$ number density $n_{\mathrm{c}} \equiv \rho_{\mathrm{c}} / \mu_{\mathrm{H}_{2}} m_{\mathrm{H}}=8 \times$ $10^{4} \mathrm{~cm}^{-3}$, flat inner radius $R_{\text {flat }}=5 \times 10^{3} \mathrm{AU}$, and outer radius

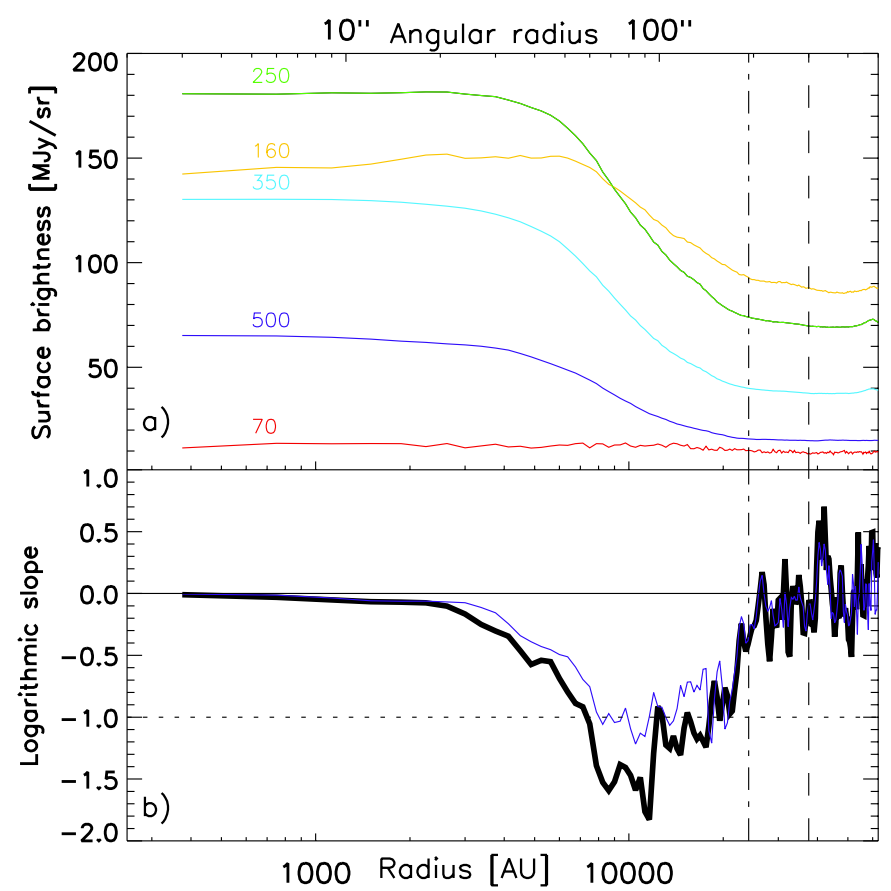

Fig. A.1. a) Circularly averaged radial surface brightness profiles of B68 at 70, 160, 250, 350, $500 \mu \mathrm{m}$ derived from Herschel/SPIRE and PACS data (after adding Planck offsets). b) Logarithmic slopes of the circularly averaged column density profile (solid black curve) and $500 \mu \mathrm{m}$ intensity profile (solid blue curve) of B68 as a function of radius. The horizontal dotted line is the logarithmic slope $s=-1$ expected for the column density profile of a core with a $\rho \propto r^{-2}$ density profile. The vertical dashed line marks the integration upper bound adopted when integrating the right-hand side of Eq. (2) to perform the Abel reconstruction of the density and temperature profiles (see Sect. 3). The vertical dot-dashed line shows the radius of the footprint automatically derived for B68 by the getsources source-finding algorithm.

$R_{\text {out }}=1.5 \times 10^{4}$ AU. The surface density profile of such a model core has an analytical form of

$\Sigma(p)=\frac{2 \rho_{\mathrm{c}} R_{\text {flat }}}{\left(1+p^{2} / R_{\text {flat }}^{2}\right)^{1 / 2}} \times \tan ^{-1}\left(\frac{\left(R_{\text {out }}^{2}-p^{2}\right)^{1 / 2}}{\left(R_{\text {flat }}^{2}+p^{2}\right)^{1 / 2}}\right)$,

where $p$ represents the impact parameter from core center in the plane of the sky and $N_{\mathrm{H}_{2}}(p)=\Sigma(p) / \mu_{\mathrm{H}_{2}} m_{\mathrm{H}}$ is the $\mathrm{H}_{2}$ column density profile. The intrinsic density profile of the model is shown as a black solid curve in Fig. B.1a and the corresponding column density profile as a black solid curve in Fig. B.1b. The synthetic dust temperature profile is shown as a black solid curve in Fig. B.1c and was obtained for a solar-neighborhood ISRF $\left(G_{0}=1\right)$ using an analytic approximation formula that reproduces a grid of spherically symmetric models performed with the dust radiative transfer code MODUST (Bouwman et al., in prep.; see Bouwman et al. 2001; and André et al. 2003).

A set of synthetic emission maps was created by line-of-sight integration of this model core at all Herschel wavelengths, assuming optically thin dust emission (see Eq. (1)) and the same dust opacity law as given in Sect. 3.1. The density and temperature profiles of the model core were then reconstructed as described in Sect. 3 from the circularly averaged radial intensity profiles of the model emission. The cross symbols overlaid on the model density, column density, and temperature profiles in Fig. B.1a-c show the Abel-reconstructed profiles that would be obtained with "infinite" angular resolution (and in the absence of 


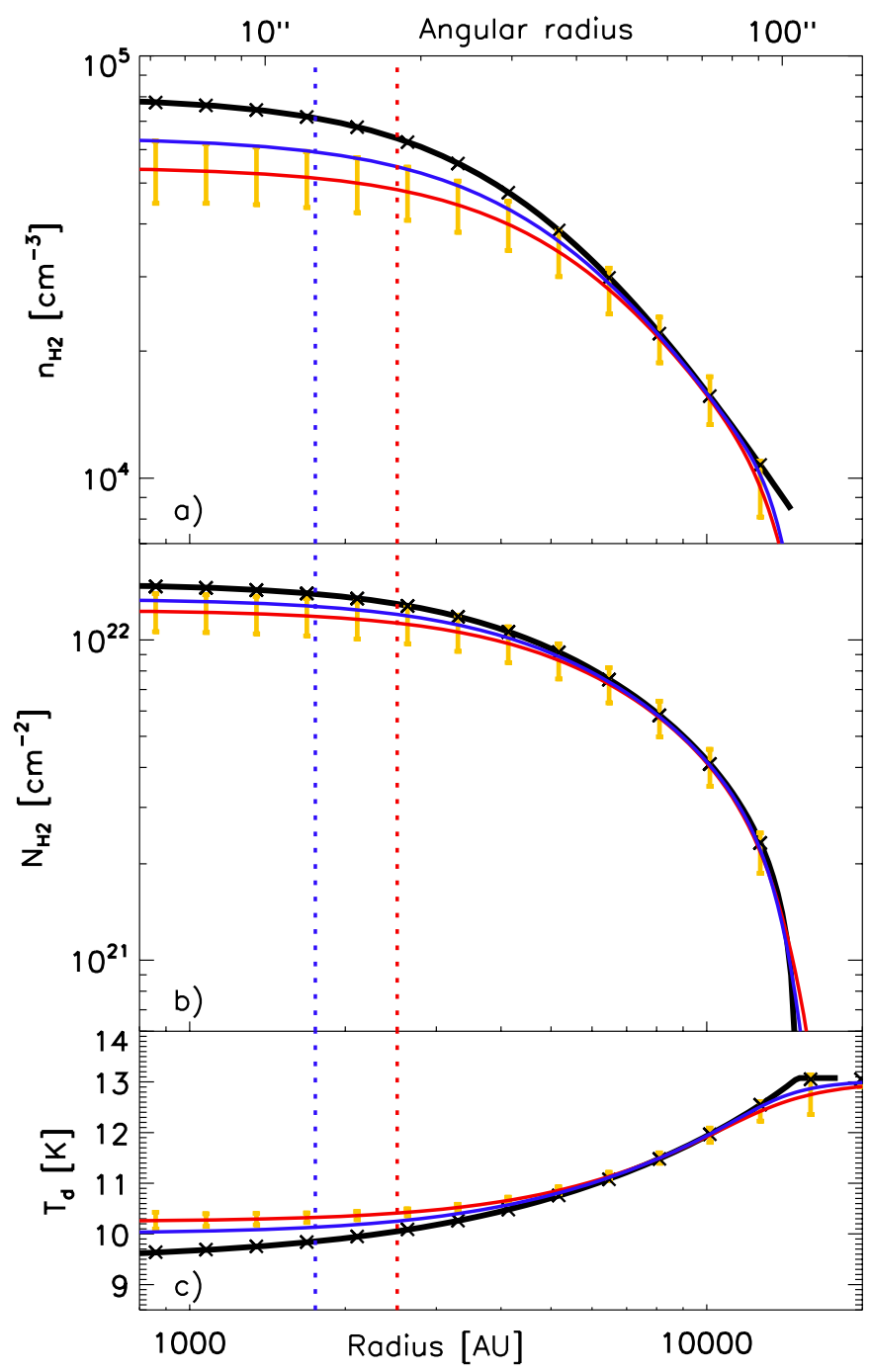

Fig. B.1. Comparison between intrinsic (black curves) and reconstructed (red and blue curves) volume density a), column density b), and dust temperature c) profiles for a spherically symmetric core model with a Plummer-like density distribution (see Eq. (B.1) and text for model parameters). The cross symbols show the results obtained by applying the Abel reconstruction scheme on the synthetic $160-500 \mu \mathrm{m}$ intensity profiles with "infinite" resolution. The red and blue curves show the reconstruction results obtained from synthetic emission maps smoothed to HPBW resolutions of $366^{\prime \prime} 3$ and 24"' 9 , respectively, corresponding to the resolutions of Herschel $500 \mu \mathrm{m}$ and $350 \mu \mathrm{m}$ observations. Note the good agreement between the reconstructed profiles and the intrinsic profiles beyond the the half power beam radius of $366^{\prime \prime} 3 / 2(500 \mu \mathrm{m}$ resolution) or $24 . ' 9 / 2$ (350 $\mu \mathrm{m}$ resolution), as marked by the red and blue vertical dotted lines, respectively. The error bars in each panel show the $1 \sigma$ uncertainties due to random noise and calibration errors.

noise). It can be seen in Fig. B.1 that, the reconstruction is perfect in this case, demonstrating the validity of our Abel-inversion code. The overplotted red curves in Fig. B.1a-c show the reconstructed volume density, column density, and temperature profiles that result from the Abel-inversion method after convolution of the model images to a common resolution of 36"' 3 . This corresponds to the Herschel resolution at $500 \mu \mathrm{m}$. Likewise, the overplotted blue curves in Fig. B.1a-c show the results obtained at a resolution of 24.9 using the synthetic data convolved to the Herschel resolution at $350 \mu \mathrm{m}$ and ignoring the $500 \mu \mathrm{m}$ data. It can be seen that the profiles reconstructed at the Herschel resolution remain in excellent $(1 \%)$ agreement with the intrinsic profiles in the outer part of the core. Although the reconstruction becomes somewhat inaccurate below the Herschel resolution limit (marked by vertical dotted lines in Fig. B.1), the reconstructed column density and temperature profiles still agree with the corresponding intrinsic profiles to within $20 \%$ and $9 \%$, respectively, at $500 \mu \mathrm{m}$ resolution. The accuracy of the results at small radii improves to $11 \%$ and $5 \%$ when the reconstruction is performed at $350 \mu \mathrm{m}$ resolution. (Although in the presence of noise with real data, the statistical measurement uncertainties are somewhat larger at $350 \mu \mathrm{m}$ resolution.) At both resolutions, the Abel-reconstructed temperature and column density profiles coincide within $1 \%$ with the corresponding intrinsic profiles that are convolved with the effective beam resolution. The reconstructed central temperature and column density thus provide excellent estimates of the beam-averaged central temperature and column density in the model. The total mass that is estimated by integrating the reconstructed column density profile agrees with the model mass more accurate than $0.1 \%$ even at $500 \mu \mathrm{m}$ resolution.

We also assessed the contribution of background fluctuations and calibration errors to the uncertainties in the derived parameters $\left(N_{\mathrm{H}_{2}}\right.$ and $\left.T_{\mathrm{d}}\right)$. To do so, we considered 500 realizations of synthetic skies, which included a random Gaussian noise component ${ }^{7}, \sigma_{v}$, and a random multiplicative calibration factor, $(1+g)$ :

$I_{v}^{\mathrm{sim}}(x, y)=\left[I_{v}^{\text {model }}(x, y)+\sigma_{v}\right](1+g)$,

where $g$ is a Gaussian random number with a mean zero and standard deviation of $10 \%$ and $15 \%$ at SPIRE and PACS wavelengths, respectively. (We assumed $100 \%$ correlated calibration errors at SPIRE wavelengths and an independent calibration error in the PACS $160 \mu \mathrm{m}$ band.) The net uncertainties in $n_{\mathrm{H}_{2}}, N_{\mathrm{H}_{2}}$, and $T_{\mathrm{d}}$ were estimated to be $15 \%, 12 \%$, and $5 \%$, respectively. The resulting $1 \sigma$ errors in the derived parameters are displayed in Fig. B.1.

\section{B.2. Ellipsoidal core model}

As real cores, such as L1689B, are often elongated and thus not strictly spherically symmetric (see Sect. 4.2), we also tested the reliability of our Abel inversion scheme using a simple nonspherical model with an ellipsoidal Plummer-like density distribution for $r \leq R_{\text {out }}$ and $z \leq Z_{\text {out }}$ with cylindrical symmetry about the $z$ axis (assumed to lie in the plane of the sky):

$\rho(r, z)=\frac{\rho_{c}}{1+\left(r / R_{\text {flat }}\right)^{2}+\left(z / Z_{\text {flat }}\right)^{2}}$,

where $R_{\text {flat }}$ and $Z_{\text {flat }}$ are the radii of the flat inner core region perpendicular and parallel to the $z$ axis of symmetry (see Fig. B.2a), respectively. We considered both the prolate $\left(Z_{\text {flat }}>R_{\text {flat }}\right)$ and the oblate $\left(Z_{\text {flat }}<R_{\text {flat }}\right)$ configuration but are primarily describing the prolate case here, as it is more likely for cores embedded within filaments such as L1689B (see Fig. 3). The synthetic temperature distribution was also assumed to be cylindrically symmetric about the $z$ axis and was constructed using the same grid of MODUST radiative transfer models as in Appendix B.1. The synthetic temperature profiles along both the $z$ axis and the radial $(r)$ direction are shown in Fig. B.2. For direct comparison

7 The level of noise fluctuations $\left(\sigma_{v}\right)$ was chosen so that the peak signal-to-noise $I_{v}^{\text {peak }} / \sigma_{v}$ value at each wavelength was consistent with the corresponding B68 surface brightness image. 

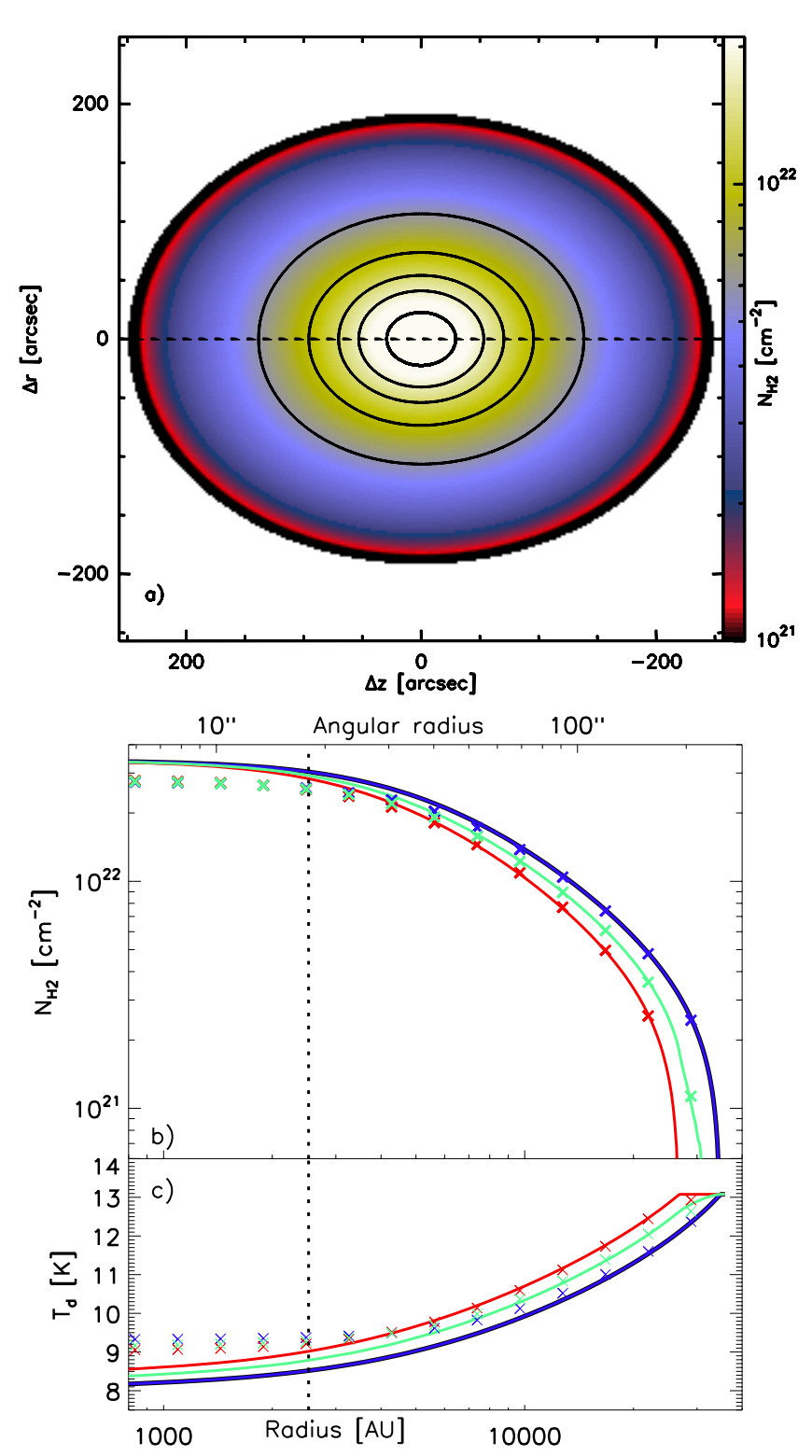

Fig. B.2. a) Synthetic column density image of a prolate ellipsoidal core model with an aspect ratio of 1.3 and a Plummer-like density distribution (see Eq. (B.4) and the text for model parameters). The horizontal dotted line shows the axis of symmetry in the plane of sky, and the contour levels are the same as in Fig. 3. b) Comparison between the intrinsic (solid curves) and the reconstructed (crosses) column density profiles of the model. c) Comparison between the intrinsic (solid curves) and the reconstructed (crosses) dust temperature profiles of the model. The blue and red curves represent the intrinsic radial profiles along and perpendicular to the axis of symmetry, respectively. The green curves represent the intrinsic circularly averaged radial profiles. The blue and red crosses display the results of the Abel-inversion method applied to the synthetic $160-500 \mu \mathrm{m}$ intensity profiles of the model, which are convolved to 36 .' 3 resolution and taken along the major and minor axes, respectively. The green crosses show the reconstruction results by using the circularly averaged intensity profiles of the model as inputs. Note the good agreement between the reconstructed profiles and the intrinsic profiles beyond the half power beam radius of $366^{\prime \prime} 3 / 2$, as marked by the vertical dotted line. with L1689B (see Figs. 3 and B.2a), we adopted physical parameters approximately consistent with the observed characteristics of the L1689B core (see Sect. 4.2): central $\mathrm{H}_{2}$ number density $n_{\mathrm{c}}=2 \times 10^{5} \mathrm{~cm}^{-3}$; aspect ratio $Z_{\text {flat }} / R_{\text {flat }}=Z_{\text {out }} / R_{\text {out }}=1.3$; flat inner radius along the minor axis $R_{\text {flat }}=4000 \mathrm{AU}$; and outer radius along the minor axis $R_{\text {out }}=6.7 \times R_{\text {flat }}=26800 \mathrm{AU}$.

Because of the lack of spherical symmetry, we applied our Abel reconstruction scheme to three sets of intensity profiles: 1) the profiles measured along the major axis of the model (intrinsic profiles shown as blue curves and results as blue crosses in Fig. B.2); 2) the profiles measured along the minor axis of the model (intrinsic profiles shown as red curves and results as red crosses in Fig. B.2); and 3) circularly averaged intensity profiles (intrinsic profiles shown as green curves and results as green crosses in Fig. B.2). Here, again, it can be seen that the reconstruction results are very satisfactory ( $2 \%$ agreement) beyond the beam radius (marked by the vertical dotted line in Fig. B.2). The reconstruction performed perpendicular to the axis of symmetry, i.e., along the minor axis for a prolate core, is more accurate $(1 \%)$ than the reconstruction performed along the axis of symmetry $(4 \%)$. In particular, the best estimate of the central dust temperature is obtained from the reconstruction performed along the minor axis. The reconstruction along the major axis nevertheless provides better estimates of the column density and temperature at large radii along the major axis. The central column density reconstructed at $500 \mu \mathrm{m}$ resolution slightly underestimates but still agrees to within $20 \%$ with the true column density at core center. The best estimate of the total core mass, as obtained by using the results of the reconstruction performed on the circularly averaged intensity profiles, agrees more accurate than $4 \%$ with the model core mass. Even for a more elongated core model with an aspect ratio of 2 (instead of 1.3), the reconstructed core mass still agrees with the model mass to within $5 \%$.

We also performed similar simulations for an oblate core model observed edge-on. The accuracy of the reconstruction results was found to be essentially the same as for the prolate case. Again, the reconstruction performed perpendicular to the axis of symmetry, i.e., along the major axis in this case, was found to be more accurate than the reconstruction performed along the axis of symmetry. The best estimate of the total core mass was again obtained from reconstructing the circularly averaged intensity profiles. 